\title{
Intubation and mechanical ventilation of patients with COVID-19: what should we tell them?
}

\author{
Nicholas Zareifopoulos $^{1}$, Maria Lagadinou ${ }^{1}$, Anastasia Karela ${ }^{1}$, Gerasimos Karantzogiannis ${ }^{1}$, \\ Dimitrios Velissaris ${ }^{1,2}$ \\ ${ }^{1}$ Emergency Department, University Hospital of Patras; ${ }^{2}$ Department of Internal Medicine, University Hospital of Patras, \\ Greece
}

\section{To the Editor}

Severe COVID-19 illness is characterised by the development of Acute Respiratory Distress Syndrome (ARDS), for which the mainstay of treatment is represented by mechanical ventilation. Mortality associated with ARDS due to other causes is in the range of $40-60 \%$, but currently available data are not yet sufficient to draw safe conclusions on the prognosis of COVID-19 patients who require mechanical ventilation. Based on data from cohorts of the related coronavirus-associated illnesses, that is to say Severe Acute Respiratory Syndrome (SARS) and Middle East Respiratory Syndrome (MERS), prognosis would seem to be worse than ARDS due to other causes such as trauma and other infections. Discussion of prognosis is central to obtaining informed consent for intubation, but in the absence of definitive data it is not clear exactly what this discussion should entail.

The COVID-19 epidemic which originated in the Hubei province of China in the autumn of 2019 has grown into a pandemic, with the United States and Southern Europe recording the highest number of cases and fatalities as of April 4, 2020. Most infected individuals develop a self-limited viral illness, with

Correspondence: Nicholas Zareifopoulos, Emergency Department, University Hospital of Patras, Ground Floor, Patras, Greece.

Tel. +30.26109999955.

E-mail: nizareifopoulos@gmail.com

Funding: There is no funding to report for this work.

Conflict of interests: The authors declare no conflict of interests.

Key words: COVID-19; SARS-CoV-2; critical care; mechanical ventilation; intubation.

Received for publication: 4 April 2020.

Accepted for publication: 6 April 2020

${ }^{\circ}$ Copyright: the Author(s), 2020

Licensee PAGEPress, Italy

Monaldi Archives for Chest Disease 2020; 90:1296

doi: 10.4081/monaldi.2020.1296

This article is distributed under the terms of the Creative Commons Attribution Noncommercial License (by-nc 4.0) which permits any noncommercial use, distribution, and reproduction in any medium, provided the original author(s) and source are credited. severe disease manifesting primarily as acute respiratory distress syndrome (ARDS). Even with optimal supportive care in the intensive care unit (ICU), many patients develop multiorgan failure and die a few days after the initiation of treatment in the ICU $[1,2]$. A retrospective cohort study of 191 patients published in the Lancet [3] sheds light on the risk factors for early mortality in patients hospitalized due to COVID-19 infection. The cohort consisted of inpatients with a definitive outcome (discharge or death) within 34 days. Such framework enables the identification of prognostic factors of early death, but the temporal restriction excludes patients with a more prolonged course of disease. The afore-mentioned patients form a heterogenous group, which we assume would consist of patients who died after a prolonged stay in the ICU, patients who required intubation but subsequently improved and survived to discharge and patients with pre-existing comorbidities or emergent complications which needed prolonged inpatient treatment. Data regarding outcomes in this group are urgently needed, as these individuals are most likely to benefit from treatment. To our knowledge, no study on the long-term outcomes of COVID-19 patients who were intubated and underwent mechanical ventilation has yet been published, though we expect that data from China may be encouraging, as the reported number of patients in critical conditions had been rapidly falling throughout the past month, without a corresponding surge in the number of deaths. An optimistic interpretation of this would see patients successfully extubated and discharged from the hospital, however cohort studies with a longer follow-up period are needed to have full confidence in treatment efficacy and outcomes.

The epidemic is at present actively spreading throughout Greece, with over 1500 confirmed cases and over 60 deaths at the time of writing. We are seriously concerned about our patients with ARDS who require mechanical ventilation, especially because the data published so far are extremely discouraging, with 32 deaths/33 patients ( $97 \%)$ who were intubated [3] in one cohort and 30/37 (81\%) in another [4]. These data clearly mean a prognosis that is worse than ARDS due to other causes, whose mortality is in the range of $40-60 \%$ with optimal disease management [5]. Only $25 \%$ of patients with Middle East Respiratory Syndrome (MERS) making up a cohort, survived to discharge [6,7], this indicates a prognosis worse than ARDS due to other causes, though it is not clear whether these findings can be generalized to COVID-19. A cohort of critically ill patients with Severe Acute Respiratory Syndrome (SARS) fared slightly better, with the survival of $15 / 39$ (38\%) patients requiring mechanical ventilation [8].

The poor prognosis of severe coronavirus-related illnesses in the setting of a pandemic, raises serious legal, ethical and health 
policy issues which have to be addressed immediately, without definitive evidence. The sorely tried medical professionals who shall choose who to intubate must be aware that they are not deciding who is going to live and who is going to die, but rather who is more likely to survive. If intubation is necessary in a COVID-19 patient, death may be a more likely outcome than survival even with optimal treatment in the ICU. The course of the disease and the possibility of intubation must be discussed with patients early in the course of their hospitalization (preferably on admission), so they can make an informed decision about the possibility to undergo mechanical ventilation. Given the low chance of recovery and the long-term complications associated with prolonged stays in the ICU, a Do Not Intubate (DNI) order would not be irrational or self-destructive, even among young healthy adults without comorbidities.

The issue with informed consent in this case is that there is no definitive answer regarding the prognosis of COVID-19 after the initiation of mechanical ventilation; therefore, should patients be informed about the outcomes of SARS, MERS and ARDS in general in order to facilitate a decision? Or would it be preferable to avoid disclosing such information (which may not be generalizable to COVID-19) and simply ask patients to consent on the basis of the fact that intubation is part of the standard of care and represents their best chance for survival if severe ARDS develops?

In any case, if patients ask, the best thing we can do is to admit what we do not know. There are thousands of patients intubated with COVID-19 worldwide, of which 92 in Greece. Only time will tell whether they will survive or not, and patients should be encouraged to accept care escalation rather than give up if it is feasible.

\section{References}

1. Wang D, Hu B, Hu C, et al. Clinical characteristics of 138 hospitalized patients with 2019 novel coronavirus-infected pneumonia in Wuhan, China. JAMA 2020;323:1061-9. doi: 10.1001/jama.2020.1585.

2. Huang C, Wang Y, Li X, et al. Clinical features of patients infected with 2019 novel coronavirus in Wuhan, China. Lancet 2020;395:497-506.

3. Zhou F, Yu T, Du R, et al. Clinical course and risk factors for mortality of adult inpatients with COVID-19 in Wuhan, China: a retrospective cohort study. Lancet 2020:395:1054-62. doi: 10.1016/S0140-6736(20)30566-3.

4. Yang X, Yu Y, Xu J, et al. Clinical course and outcomes of critically ill patients with SARS-CoV-2 pneumonia in Wuhan, China: a single-centered, retrospective, observational study. Lancet Respir Med 2020. doi: 10.1016/S2213-2600(20)30079-5.

5. Bellani G, Laffey JG, Pham T, et al. Epidemiology, patterns of care, and mortality for patients with acute respiratory distress syndrome in intensive care units in 50 countries. JAMA 2016;315:788-800.

6. Alraddadi BM, Qushmaq I, Al-Hameed FM, et al. Noninvasive ventilation in critically ill patients with the Middle East respiratory syndrome. Influenza Other Respi Viruses 2019;13:382-90.

7 Arabi YM, Al-Omari A, Mandourah Y, et al. Critically ill patients with the Middle East respiratory syndrome: A multicenter retrospective cohort study. Crit Care Med 2017;45:1683-95.

8. Lew TWK, Kwek T-K, Tai D, et al. Acute respiratory distress syndrome in critically ill patients with severe acute respiratory syndrome. JAMA 2003;290:374-80. 